\title{
Linx
}

Revue des linguistes de l'université Paris X Nanterre

$79 \mid 2019$

Enseigner et apprendre à interagir en langue étrangère : réflexions linguistiques et didactiques

\section{Pourquoi faire de la sociolinguistique des interactions verbales avec des enseignants et des apprenants de Français Langue Étrangère?}

On the usefulness of sociolinguistic analysis of interactions for learners of French as a Foreign Language

\section{Virginie ANDRÉ}

\section{CpenEdition}

Journals

Édition électronique

URL : http://journals.openedition.org/linx/3694

DOI : $10.4000 / \operatorname{linx} .3694$

ISSN : 2118-9692

Éditeur

Presses universitaires de Paris Nanterre

Référence électronique

Virginie ANDRÉ, « Pourquoi faire de la sociolinguistique des interactions verbales avec des enseignants et des apprenants de Français Langue Étrangère ? », Linx [En ligne], 79 | 2019, document 7, mis en ligne le 02 mars 2020, consulté le 06 avril 2020. URL : http://journals.openedition.org/linx/ 3694 ; DOI : https://doi.org/10.4000/linx.3694

Ce document a été généré automatiquement le 6 avril 2020.

Département de Sciences du langage, Université Paris Ouest 


\section{Pourquoi faire de la sociolinguistique des interactions verbales avec des enseignants et des apprenants de Français Langue Étrangère?}

On the usefulness of sociolinguistic analysis of interactions for learners of French as a Foreign Language

\section{Virginie ANDRÉ}

\section{Introduction}

Nous proposons une exploitation didactique de l'analyse sociolinguistique des interactions verbales. La description des interactions en français est encore parcellaire et n'est que trop peu diffusée en didactique des langues. D'une part, les analyses interactionnelles méritent d'être approfondies afin d'améliorer les connaissances sur la façon dont les locuteurs interagissent et notamment sur les ressources qu'ils utilisent pour interagir de façon satisfaisante, c'est-à-dire pour accomplir leurs objectifs dans et en dehors de l'interaction. D'autre part, ces connaissances méritent d'être diffusées auprès des enseignants et des apprenants de Français Langue Étrangère (désormais FLE). Elles permettent de proposer des programmes de formations appropriés, en lien avec la réalité, la complexité et la dynamique des interactions verbales. Cet article s'articule en deux principales parties. Chacune montre l'importance d'exploiter les résultats des recherches sur les interactions verbales en didactique afin d'enseigner et d'apprendre une langue réelle, c'est-à-dire la variété des usages en situation. Tout d'abord, nous exposerons le cadre sociolinguistique qui permet d'analyser les interactions et de pointer des phénomènes interactionnels intéressants pour l'enseignement et l'apprentissage du français. Nous présenterons une analyse des 
questions et du marqueur genre dans le corpus $\mathrm{TCOF}^{1}$. Nous verrons que les résultats de ces analyses sont rarement abordés en formation linguistique. Ensuite, nous proposerons d'autres exploitations didactiques en exposant deux façons complémentaires - d'utiliser des corpus d'interactions : en analysant le déroulement et les spécificités des interactions, conjointement entre enseignant et apprenant, ou en interrogeant le corpus avec un concordancier. Ces deux façons de faire de la sociolinguistique avec des enseignants et des apprenants seront illustrées avec le dispositif FLEURON (Français Langue Étrangère Universitaire: Ressources et Outils Numériques) ${ }^{2}$ qui met à disposition un corpus multimodal de situations de communication authentiques vécues par des étudiants. Il permet ainsi à des étudiants étrangers de se préparer à interagir dans les situations qu'ils vont rencontrer en arrivant en France ou lors de leur séjour universitaire (André 2016). Le corpus est outillé d'un concordancier qui permet aux apprenants de faire des requêtes et d'analyser les résultats, selon la méthodologie du data-driven learning (Johns 1991). En proposant aux apprenants des interactions verbales authentiques situées à observer et à analyser, ils apprennent à interagir de façon appropriée. Ce dispositif nous permet ainsi d'illustrer l'importance d'établir des liens entre sociolinguistique et didactique.

\section{Les apports de la sociolinguistique des interactions verbales}

2 La complexité des interactions verbales doit faire appel à un cadre d'analyse qui permet de prendre en compte les multiples intrications et influences qui orientent et qui pèsent sur le déroulement des échanges. L'analyse sociolinguistique des interactions verbales met en synergie différentes approches et disciplines ainsi que différents concepts et outils afin de saisir les pratiques interactionnelles des locuteurs et les activités interactionnelles qu'ils réalisent lorsqu'ils sont engagés dans des genres de discours et des situations de communication particuliers. Le schéma suivant illustre les intrications et les influences mentionnées précédemment. Il peut être utilisé comme un moyen mnémotechnique pour déterminer l'ensemble des éléments à étudier lorsque l'on souhaite analyser des interactions verbales.

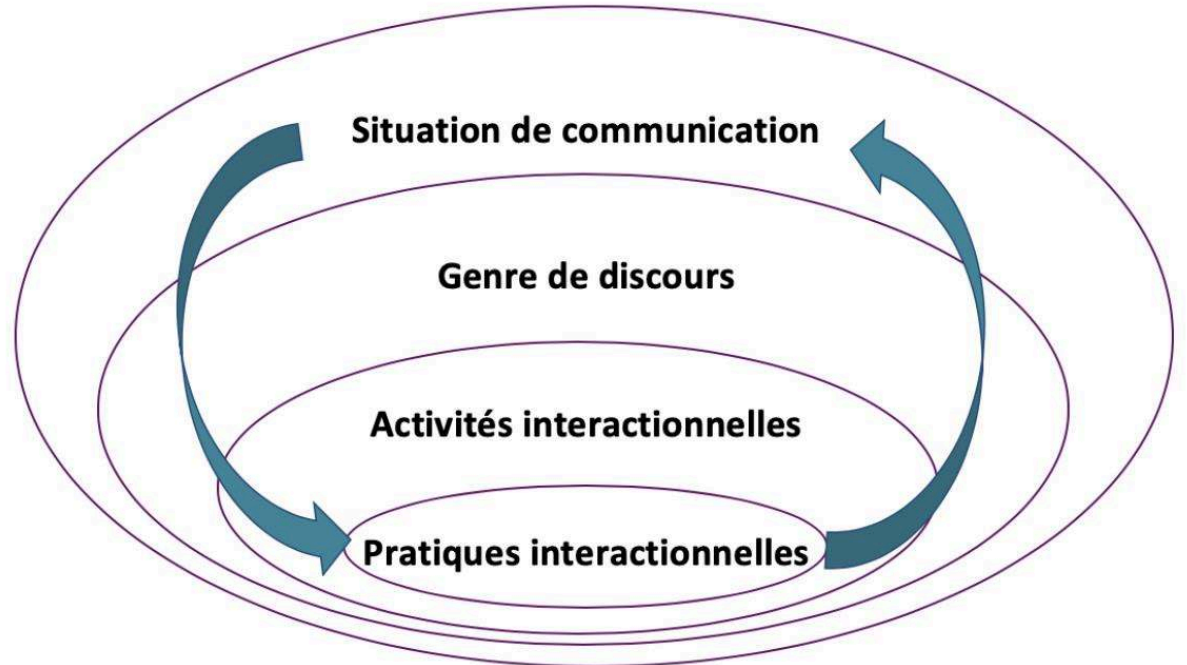

Figure 1 : Intrications et interinfluences dans les interactions verbales (André 2019 : 18). 
Nous pouvons décrire ce schéma en partant de son centre, c'est-à-dire du matériel interactionnel à analyser ou encore des pratiques interactionnelles. Ces dernières sont les ressources langagières et non langagières (verbales et non verbales) mises en œuvre par les locuteurs pour interagir. Ces ressources sont infinies et variées. Elles sont caractérisées et particularisées par le lexique, les constructions syntaxiques, les répétitions, les reformulations, les marqueurs de discours, les ponctuants spécifiques à l'oral, la prosodie, les interjections, les bruits-sons (pff, $\mathrm{mh}$, tss), les gestes et les postures, les regards et les expressions faciales. Elles prennent sens dans la temporalité de l'interaction, c'est-à-dire au fur et à mesure de leur apparition dans l'échange, et configurent l'interaction. Comme toutes les pratiques sociales, elles s'inscrivent dans une situation. Elles accomplissent des activités sociales et interactionnelles. Ces dernières sont de différentes natures. Elles correspondent à des activités telles que poser des questions, s'excuser, expliquer, raconter une histoire, complimenter, informer, reprocher, convaincre, saluer, remercier, inviter, insulter, etc. Leurs analyses héritent des théories traditionnelles et pragmatiques des actes de langages (Austin 1962, Searle 1969). Ces mêmes activités interactionnelles peuvent être accomplies avec plusieurs activités différentes. Par exemple, convaincre son interlocuteur est une activité interactionnelle mais elle peut être accomplie avec plusieurs autres activités telles qu'argumenter, expliquer, illustrer, etc. Dans ce cas, une macro-activité est accomplie grâce à plusieurs micro-activités (Kerbrat-Orecchioni 2001). Les différentes activités interactionnelles peuvent donc être entremêlées pour accomplir les objectifs de la communication. Les activités interactionnelles structurent également les interactions lorsqu'elles ouvrent les échanges, permettent de changer de thème, de prendre la parole, d'interrompre son interlocuteur, de le compléter ou encore de clore les échanges (Traverso 2016). Les activités interactionnelles ont des effets pragmatiques, c'est-à-dire des incidences ou des conséquences sur les interactions et sur la situation de communication. Ces effets ne sont pas toujours ceux escomptés par les locuteurs. Ils peuvent être négociés, acceptés ou réfutés.

4 Le genre de discours correspond au type d'interaction, au speech event (Hymes 1972) ou encore selon Kerbrat-Orecchioni et Traverso (2004: 42) au « G1 » qui est « un ensemble discursif plus ou moins institutionnalisé dans une société donnée ». Il est défini par des critères extralinguistiques, tels que le cadre situationnel, le cadre participatif, le degré de formalité ou l'objectif de l'échange. Dans notre cadre d'analyse, les genres de discours sont par exemple : des conversations, des réunions de travail, des entretiens, des débats, des conférences, des cours, des relations transactionnelles, etc. Ils sont constitués d'activités interactionnelles. En d'autres termes, ces « G1 sont composés de G2 », selon la terminologie de Kerbrat-Orecchioni et Traverso (2004 : 44), c'est-à-dire composés d'activités interactionnelles. La situation de communication est constituée de l'ensemble des éléments extralinguistiques qui conditionnent le déroulement de l'interaction. Il s'agit de décrire de façon ethnographique la situation (Hymes 1972) dans laquelle prend place une interaction. La situation est composée de tous les éléments qui préexistent à l'interaction. Par exemple, la notion de participants comprend de multiples éléments, tels que le cadre participatif, le nombre de locuteurs, l'identité des locuteurs, leurs statuts, fonctions, rôles sociaux et conversationnels, leurs relations interpersonnelles et hiérarchiques, mais également leur histoire conversationnelle (Golopentia 1988). Les situations de communication ont une épaisseur historique (Boutet 1994), c'est-à-dire qu'elles s'inscrivent dans une histoire 
sociale, économique, culturelle particulière. La description des éléments de la situation de communication permet de s'interroger sur leur influence en interaction. La situation et ses éléments peuvent également être redéfinis et modifiés par l'interaction.

Ce schéma a pour objectif de représenter les multiples intrications et influences en jeu dans les interactions verbales. Les cercles représentent ces intrications, chacun des éléments du schéma est lié aux autres. Les flèches symbolisent les influences traversant ces quatre cercles. Plus précisément, les flèches illustrent la réciprocité des influences, de la situation de communication vers les pratiques interactionnelles et des pratiques vers la situation. Ainsi, les pratiques interactionnelles sont toujours situées, influencées par les éléments de la situation et, de façon réflexive (Mondada 2006), transforment certains de ces éléments. Ce cadre d'analyse permet de saisir la complexité des interactions verbales et de comprendre le fonctionnement du français parlé en interaction. Nous allons illustrer cette complexité en analysant deux pratiques interactionnelles : les questions et le marqueur genre. Nous verrons que les éléments du schéma-cadre doivent être sollicités pour l'analyse de ces pratiques.

\subsection{Les questions}

6 Parmi les pratiques interactionnelles aux prises avec de multiples influences, il est intéressant d'examiner les questions en interaction. L'objectif de cette étude n'est pas de proposer une analyse exhaustive des différentes formes de questions (voir notamment Kerbrat-Orecchioni 1991 ; Coveney 1996 ; Rossari et al. 2005 ; Beguelin et al. 2018) mais d'identifier en quoi l'analyse sociolinguistique des interactions verbales permet de pointer des phénomènes socio-interactionnels spécifiques, aux prises avec de multiples influences. Nous n'analyserons pas les questions dont les formes sont bien identifiées et bien décrites dans les documents de références (grammaires et manuels) ou dans de nombreux travaux linguistiques. Ces formes sont: l'inversion sujet-verbe, l'utilisation d'un mot interrogatif ou de « est-ce que » et l'assertion avec une intonation montante ou ouvrante. Nous nous intéresserons ici aux questions dont les formes sont peu voire ne sont pas décrites, mais qui apparaissent fréquemment dans les interactions. Nous nous concentrerons sur les questions qui prennent la forme d'un énoncé inachevé ou suspensif (Debaisieux, \& Boulton 2007). Nous analyserons les conditions d'apparition de ces questions spécifiques ainsi que leurs effets pragmatiques dans les interactions. Pour réaliser cette étude, nous avons analysé la partie adulte du corpus $\mathrm{TCOF}^{3}$. Cette partie compte actuellement 253 enregistrements d'une durée totale de 60 heures et est évalué à 760000 mots transcrits ${ }^{4}$.

7 Parmi les énoncés syntaxiquement inachevés ou suspendus qui permettent de poser des questions, les énoncés alternatifs ne mentionnant qu'une seule des propositions sont relativement fréquents. Ils s'achèvent généralement par un allongement sur la conjonction de coordination ou, comme c'est le cas dans l'exemple qui suit.

Exemple 1 : Lors d'un entretien de type sociologique, $\mathrm{L}^{5}$ interroge $\mathrm{L} 2$ sur son anorexie (Anorexie_BAN_17).

L2 ça a duré vraiment on va dire médicalement parlant avec un suivi et un arrêt de travail pendant presque deux ans pendant deux ans je ne suis pas retournée travailler

L1 et quand tu dis que tu as eu un suivi tu as eu un suivi euh avec un médecin euh généraliste plutôt comme ça ou:: 
L2 au départ au départ c'était généraliste mais il a vite été dépassé par la situation certains éléments de la situation de communication. Les locuteurs ne se connaissent pas et le sujet de l'échange est personnel et potentiellement délicat. L1 souhaite demander si L2 a été suivie par un médecin ou par un spécialiste, par exemple un psychologue. Cependant, afin de préserver la face de son interlocutrice (Goffman 1974), L1 choisit de poser une question alternative sans proposer d'alternative à «médecin généraliste » qui ne risque que peu de mettre mal à l'aise L2, puisqu'elle vient de dire qu'elle a eu un traitement et qu'elle a été suivie. L'allongement produit sur la conjonction ou (symbolisé par ::) peut être interprété comme un choix de ne pas proposer un achèvement syntaxique à cet énoncé et de ne pas proposer une ou plusieurs alternatives. Toutefois, cet énoncé est bien achevé pragmatiquement, l'activité interactionnelle est réalisée, et $\mathrm{L} 2$ prend le tour pour répondre à la question posée en commençant par «au départ... » (58 secondes après cette réponse, L2 dit qu'elle est allée voir un psychologue).

9 Dans d'autres situations, ce procédé apparait lorsque le locuteur ne sait pas quelle alternative il pourrait proposer ou lorsque que l'alternative est la négation de la première proposition. Il est parfois difficile de se prononcer pour l'une ou l'autre des analyses, comme c'est le cas dans l'exemple suivant:

Exemple 2 : Quatre amies discutent de cirque, que pratique L1. L'extrait porte sur le nom donné à une école de cirque (Cirque_HEL_17).

L1 mais il aurait pu à mon sens ça aurait été plus juste d'appeler ça l'académie

Gachet que de garder le nom Fratellini < parce que

L3 hum hum > ça n'avait rien à voir

L4 mais il l'a gardé

L1 d'une certaine manière non mais ça avait à voir parce que c'était du cirque

L4 il l'a gardé par hommage ou:

L1 il l'a gardé parce que c'est Paul Fratellini qui est venu le chercher pour < essayer

L2 ouais >

L1 de sauver l'école d'Annie Fratellini et que la l'école d'Annie Fratellini est devenue l'académie Fratellini avec un tout autre projet dans d'autres locaux

Après que $\mathrm{L} 1$ a donné son avis sur le nom d'une école de cirque, L4 demande une précision sur les raisons de ce choix avec l'énoncé alternatif, syntaxiquement inachevé, il l'a gardé par hommage ou (en gras). Il semble que L4 n'ait pas d'alternative à proposer, ce qui explique l'allongement sur la conjonction ou. L4 fait une première proposition et laisse une place de transition pour que L1 réponde, ce qui se produit immédiatement.

11 Les énoncés suspensifs permettant de poser des questions peuvent parfois se terminer par une hésitation allongée, c'est-à-dire un euh accompagné d'un allongement. L'exemple suivant illustre ce procédé.

Exemple 3 : Trois amies échangent à propos d'une association qui accueille des jeunes migrants (souvent des mineurs isolés). L1 est bénévole dans cette association et apporte son témoignage à ses amies.

L3 ouais enfin comment euh comment ça se fait qu'ils soient arrivés ici à Nancy et pas ailleurs ou enfin où sont leurs parents ou enfin (...) je sais pas c'est peut-être une question bête mais

L1 il y en a partout en France hein

L3 ouais

L1 ils viennent de partout (...) 
L2 c'est par rapport aux places euh::

L1 ouais c'est ça interlocuteur et d'obtenir des informations. Ils permettent de produire des énoncés stratégiques, soit en termes de préservation de la face de son interlocuteur ou de la sienne, soit en termes de méconnaissances du domaine abordé, soit les deux. Au-delà de l'objectif scientifique qui consiste à affiner la description du français parlé en interaction, les résultats de ces analyses peuvent contribuer à améliorer l'enseignement et l'apprentissage $d u$ FLE. L'observation de ces pratiques interactionnelles situées peut notamment aider les apprenants à les comprendre puis à les utiliser en situation.

\subsection{Le marqueur genre}

L'analyse sociolinguistique des interactions verbales permet de saisir le sens, les valeurs et les effets pragmatiques des régulateurs ou des marqueurs, tels que genre. Plusieurs études ont déjà été réalisées sur le marqueur genre, au-delà de ses utilisations dans des expressions telles que : un genre de, du genre à ou encore ce genre de chose. Yaguello (1998 : 18) remarquait il y a un peu plus de vingt ans que cette "nouvelle particule » se répandait « de plus en plus dans la génération de [ses] enfants ». Depuis, les valeurs pragmatiques de genre ont été identifiées. Il sert à exemplifier, illustrer, apporter une preuve, quantifier, introduire une explication, introduire un discours rapporté, catégoriser (Yaguello 1998, Fleischman 1998, Rosier 2002, Danon-Boileau \& Morel 1998, Dostie \& Pusch 2007, Vladimirska 2016). Il permet également de jouer un rôle dans la construction syntaxique d'un énoncé (Rosier 2002, Chauveau-Thoumelin 2016), il peut remplacer comme si ou une conjonction de subordination. Il peut également introduire un discours métalinguistique (et serait l'équivalent d'une unité 
telle que je veux dire par là). Et enfin, il est également un marqueur interactionnel, c'està-dire qu'il joue un rôle au niveau de l'interaction et de l'implication de son interlocuteur dans son discours.

Nous avons analysé les fonctionnements du marqueur genre au sein du corpus $\mathrm{TCOF}^{7}$. Nous avons identifié huit catégories illustrant ses différentes valeurs pragmatiques et interactionnelles. Nous les présentons brièvement.

\section{(1) genre : pour illustrer, exemplifier, apporter une preuve}

Les valeurs pragmatiques du marqueur genre les plus facilement identifiables sont celles qui consistent à illustrer, exemplifier ou apporter une preuve. Ces utilisations du marqueur sont les plus fréquentes et représentent les premières utilisations de genre sans qu'il fasse partie d'une expression plus longue telle que " un genre de ».

Exemple 1. Deux étudiantes échangent sur leur avenir professionnel et ce qu'elles envisagent de faire après leurs études. (Corpus_AMA_15)

L1 tu veux faire quoi toi

L2 moi je veux faire quelque chose en rapport avec la langue des signes + genre

accompagner les enfants sourds (...)

L1 et genre orthophoniste ça te plairait pas /

19 L2 donne un exemple de ce qu'elle souhaite faire après ses études, elle introduit cet exemple (accompagner les enfants sourds) avec genre. Ensuite, L1 propose un autre exemple (orthophoniste) qu'elle interroge avec ça te plairait pas qui présente une intonation montante (marquée par /).

\section{(2) genre : pour donner une approximation}

20 Parmi ses valeurs pragmatiques également facilement identifiables, genre permet de donner une approximation. Dans ces cas, genre est souvent synonyme de « environ».

Exemple 2. Un jeune homme raconte son voyage en Angleterre. (Coree_GHU_14)

L1 sur un site ouais on était parti avec les enfants à Worthing qui est à peu près à genre une heure de Brighton enfin de Ovingdean

21 Dans cet exemple, L1 donne une approximation de la distance en temps entre deux villes.

\section{(3) genre : pour introduire une explication}

22 Le marqueur genre permet d'introduire une séquence explicative ou d'apporter des explications sur ce qui est en train d'être dit. Il introduit souvent une parenthèse explicative qui permet à l'interlocuteur de mieux saisir ce que le locuteur en place est en train de dire. Ce dernier peut se rendre compte qu'il manque des éléments à son interlocuteur pour comprendre ses propos. Il insère alors une explication avec genre.

Exemple 3. Un jeune homme raconte à ses amis son travail en Australie. (Garcons_TOU_17)

L2 mais ouais ouais quand même là-dessus j'ai eu de la chance [rire] [toux] mais c'était chaud parce que genre euh dans la ferme de kiwis tu sais c'était pendant

l'été

L3 ouais 
L2 il faisait trente-cinq degrés et on avait ramassé des kiwis sous trente-cinq degrés euh toute la journée lors de son travail à la ferme. Le locuteur produit «c'était chaud » puis il explique que c'est parce que c'était l'été. La suite de l'interaction portera sur les conditions de travail difficiles que $\mathrm{L} 2$ a rencontrées.

\section{(4) genre : pour catégoriser}

$$
\text { permet de catégoriser et qui fonctionne de la même façon que « une espèce de ». }
$$

Exemple 4. Une jeune femme raconte son voyage en Inde. (Inde_BEL_ZIN_15)

L1 c'est vraiment une des choses qui nous a le plus euh le plus surpris et au niveau de la circulation eh bien il y a des voitures des taxis mais il y a tout il y a des vélos il $\mathrm{y}$ a des tuks-tuks ces petites mobylettes avec des places pour transporter genre taxis mais il y a aussi des animaux il y a des euh comment des chameaux des dromadaires qui tirent des charrettes

Le locuteur explique qu'il existe de nombreux moyens de transport qui peuvent s'apparenter à des taxis. Il introduit la catégorie « taxi » avec genre.

\section{(5) genre : pour introduire un discours indirect ou une scène recréée}

Dans d'autres cas, le marqueur genre sert à introduire un discours rapporté ou à présenter une scène ou un événement. Le marqueur permet de faire une transition entre les paroles du locuteur et celles qu'il rapporte ou celles qui décrivent une situation vécue.

Exemple 5. Un jeune homme raconte à ses amis son travail en Australie. (Garcons_TOU_17)

L2 je devais quitter la famille d'accueil j'avais nulle part où aller j'avais pas de taff et tout tu vois et genre euh ben à minuit moins dix j'ai reçu un mail de de la ferme qui me disait oh ben c'est bon si vous voulez vous pouvez venir euh demain on vous loge

L2 introduit la description du mail par genre. Il rapporte le contenu du message écrit qu'il a reçu de la part de la ferme.

\section{(6) genre : pour impliquer son interlocuteur dans son discours}

28 La valeur la plus récente du marqueur genre est probablement sa valeur interactionnelle, celle qui permet d'introduire son interlocuteur dans son discours. En effet, depuis une dizaine d'années, genre est utilisé très fréquemment dans les interactions par des locuteurs âgés entre 15 et 25 ans avec cette valeur implicative. Elle est parfois identique au marqueur « tu vois » ou « tu sais ».

Exemple 6. Une jeune femme raconte un film à un ami. (Film_TRE_15)

L1 ouais bah j- c'est ça qui rythme le truc que là genre il y en a un c'est un acteur et l'autre c'est un prof d'histoire euh à la fac je crois donc euh en gros il y en a qui a une vie de ouf l'autre qui a une vie de merde et euh et oui c'est le même principe en fait c'est un schizo tu vois mais il y a des trucs trop bizarres avec une histoire 
d'araignée pas compris pourquoi genre euh genre la fin et vraiment euh genre sa copine est enceinte enfin sa femme enfin bref elle est enceinte et genre euh tout à

la fin il va la voir dans une pièce et en fait elle s'est transformée en araignée corpus TCOF est celle d'introducteur de question. Ce marqueur est parfois utilisé pour introduire des alternatives telles que « tu préfères genre salé ou genre sucré ».

\section{Exemple 8.1. Une jeune femme (L1) interroge son ami (L2) sur son ancien métier de militaire. (Armee_MER_15)}

L2 bah nous on a ses rations combat et on dort euh + sur des pauvres lits de merde et juste avec une petite moustiquaire au-dessus pour dire que on est protégé quoi

L1 mais genre c'est parce qu'ils ont pas les budgets ou euh

L2 oui l'armée française n'a pas du tout le budget

L2 explique les conditions dans lesquelles se trouvent les militaires français. L1 introduit une question par genre.

Toutes ces analyses sont présentées brièvement mais pour chaque exemple les éléments de la situation de communication ainsi que le genre de discours ont été sollicités pour saisir l'apparition de cette pratique et les activités interactionnelles qu'elle peut accomplir. Nous pouvons remarquer que le marqueur genre est nettement plus utilisé par des locuteurs jeunes engagés dans des conversations informelles. Contrairement à " du coup " par exemple, qui est utilisé quelle que soit l'identité des locuteurs et les genres de discours, genre est plus restreint et contraint par les éléments situationnels. Les analyses que permet de réaliser la sociolinguistique des interactions verbales sont indéniablement utiles à la didactique du FLE. Ces analyses ne sont présentes dans aucun manuel et dans aucune grammaire. Cependant, les pratiques interactionnelles analysées sont utilisées par les locuteurs en situation de communication et sont souvent à l'origine de malentendus ou d'incompréhension de la part des apprenants de FLE. L'apport de ces analyses, pour les enseignants et les apprenants, est conséquemment une sensibilisation à la variation. 


\section{Exploitations didactiques et FLEURON}

L'objectif de cet article est de montrer que les analyses sociolinguistiques des interactions verbales peuvent être exploitées à des fins didactiques. Il s'agit de transposer les résultats des analyses interactionnelles pour les besoins de l'enseignement et de l'apprentissage du français parlé en interaction. Les manuels de FLE ne proposent que rarement des interactions authentiques aux apprenants, comprenant les spécificités que peut relever l'analyse sociolinguistique des interactions ainsi que leur variation (Giroud, Surcouf 2016). Si les manuels ne proposent que peu d'interactions authentiques, c'est probablement parce que les données manquent ainsi que des formations à les utiliser. Les enseignants de FLE le mentionnent régulièrement lorsque nous proposons des expérimentations de FLEURON. Pour pallier ce manque de corpus, plusieurs initiatives sont apparues ces dernières années. Nous pouvons tout d'abord citer CLAPI-FLE ${ }^{8}$ qui met à disposition des données multimodales traitées et didactisées pour des enseignants (voir Ravazzolo et Etienne dans ce même numéro ou Alberdi, Étienne, Juin-Chardon 2018). Ensuite, les ressources FLORALE 9 (Surcouf, Ausoni 2018) ou PFC-EF ${ }^{10}$ (Detey et al. 2009, Detey et al. 2010) sont également créées afin de proposer des données orales authentiques. Enfin, le dispositif FLEURON met à disposition des enseignants et des apprenants un corpus multimodal interrogeable par un concordancier. C'est ce dispositif que nous utiliserons pour illustrer notre démarche.

\subsection{Analyse conjointe - enseignant/apprenant - des interactions verbales}

Pour un enseignant, la première façon d'exploiter les résultats des analyses interactionnelles est de réaliser ce type d'analyse avec des apprenants. L'objectif est de pointer au fur et à mesure de la temporalité des interactions la façon dont celles-ci se déroulent. Ainsi, tour de parole par tour de parole, les enseignants peuvent aider les apprenants à saisir la façon dont les locuteurs interagissent, la façon dont les activités interactionnelles sont réalisées et les influences du genre de discours et de la situation de communication.

Nous allons examiner un exemple avec une vidéo de FLEURON intitulée « Paiement en espèces " (Figure 2). Cette vidéo présente une interaction entre un agent administratif de la scolarité (A) et une étudiante (E) qui veut régler une partie de son inscription mais qui rencontre des problèmes pour effectuer le paiement car elle n'a pas d'espèces. 


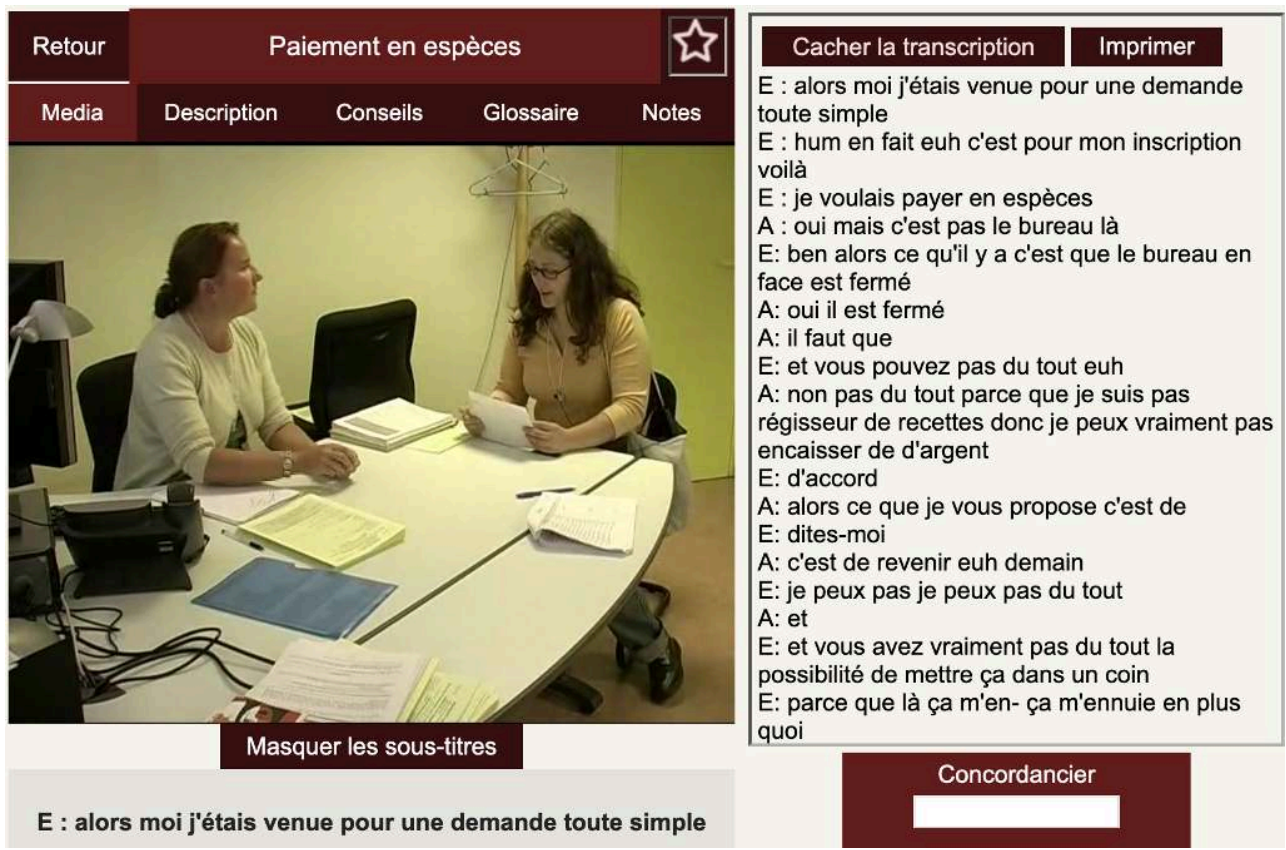

Figure 2 : Début de l'interaction de la ressource « Paiement en espèces » dans FLEURON

Ce qui est proposé avec cette ressource et ce qui a été expérimenté avec des apprenants de français à l'université, c'est une analyse sociolinguistique réalisée conjointement entre l'enseignant et l'apprenant. Concrètement, tour de parole par tour de parole, l'enseignant peut pointer les spécificités du français parlé en interaction (pas toutes pour ne pas surcharger cognitivement l'apprenant et pour s'adapter à l'apprenant). Pour illustrer cette démarche, nous allons pointer plusieurs points de cette interaction, tel que nous l'avons fait lors d'expérimentations avec des étudiants étrangers (généralement autour du niveau B1 du CECRL).

Tout d'abord, l'observation des métadonnées de la ressource indiquent que la situation de communication est assez formelle, entre des locutrices qui ne se connaissent pas et qui se vouvoient lors d'une relation professionnelle. Après ces premières observations, l'analyse de la progression de l'interaction peut commencer. Pour faciliter la lecture, nous reproduisons la transcription de l'interaction analysée.

\section{Extrait de la transcription de « Paiement en espèces » (FLEURON)}

1. E : alors moi j'étais venue pour une demande toute simple hum en fait euh c'est pour mon inscription voilà je voulais payer en espèces

2. A : oui mais c'est pas le bureau là

3. $\mathrm{E}$ : ben alors ce qu'il y a c'est que le bureau en face est fermé

4. A : oui il est fermé il faut que

5. E : et vous pouvez pas du tout euh

6. A : non pas du tout parce que je suis pas régisseur de recettes donc je peux vraiment pas encaisser de d'argent

7. E : d'accord

8. A : alors ce que je vous propose c'est de

9. $\mathrm{E}$ : dites-moi

10. A : c'est de revenir euh demain

11. E : je peux pas je peux pas du tout

12. A : et 
13. E : et vous avez vraiment pas du tout la possibilité de mettre ça dans un coin parce que là ça m'en- ça m'ennuie en plus quoi

Nous proposons ci-dessous une liste des éléments qui peuvent être pointés par un enseignant lors de l'analyse de cette interaction avec un apprenant. Cette démarche est permise grâce aux résultats de l'analyse des interactions tels que ceux que nous avons présentés dans la première partie de cet article. Nous examinons, pour l'exemple cidessus, seulement les deux premiers tours de parole.

Tour 1 : alors moi j'étais venue pour une demande toute simple hum en fait euh c'est pour mon inscription voilà je voulais payer en espèces

L'étudiante (E) commence son tour par alors, ce petit mot de l'oral permet de prendre la parole, il est également utilisé par l'agent (A) pour commencer son tour (ligne 8). Il est facile de faire remarquer à un apprenant (qui possède un métalangage) que cet adverbe, selon les grammaires, est détourné à l'oral, y compris dans une situation plutôt formelle et entre des locutrices qui ne se connaissent pas.

Il est également possible de pointer la dislocation moi je qui apparait dans cette situation plutôt formelle alors qu'elle est exclue des manuels et stigmatisée par les grammaires.

Ensuite, il est intéressant de montrer à des apprenants que la locutrice utilise un temps du passé, le plus-que-parfait, pour expliquer sa présence actuelle et sa demande future avec j'étais venue. Lors de chaque utilisation de cette ressource, les apprenants sont surpris par cette pratique, puis comprennent son utilisation.

La raison de la venue est une demande. La locutrice attenue cette demande en la qualifiant de toute simple.

Ensuite, la locutrice expose le thème de la raison de sa venue hum en fait euh c'est pour mon inscription voilà. Cette unité est composée d'un noyau thématique « c'est pour mon inscription ", de petits mots de l'oral " hum », " en fait ", " voilà » et d'une hésitation. Cette façon d'exposer un élément, avec différents marqueurs, ne fait - (presque) évidemment - pas partie de la langue ou de l'interlangue des apprenants mais elle ne fait pas non plus partie des énoncés qu'ils comprennent facilement, même au niveau B1. Dans ce tour, hum en fait euh sert de pivot entre la préparation de la demande et l'exposé du thème de la demande.

La demande est exposée en fin de tour après la transition marquée par voilà.

La locutrice produit de nouveau un verbe au passé - à l'imparfait - pour exprimer une action future : je voulais payer en espèces, ce qui représente la raison de sa venue. On constate ici l'utilisation de l'imparfait de politesse.

En bref, ce tour est composé de plusieurs unités qui posent, au fur et à mesure de leur apparition, la raison de la venue de l'étudiante. Ce tour sert ici d'ouverture de l'interaction.

\section{Tour 2 : oui mais c'est pas le bureau là}

L'agent répond en commençant son tour par « oui mais ». Dans d'autres études, nous avons analysé les mouvements effectués par « oui non mais » en début de tour (André 2005, 2010). Ces études peuvent être réinvesties pour analyser cet énoncé. L'agent montre à l'étudiante qu'elle a bien entendu sa requête avec « oui » puis elle introduit avec « mais » l'argument qui va la conduire à la refuser. Faire observer cet énoncé par des apprenants ne signifie pas que ceux-ci doivent être capables de le produire mais, dans un premier temps, ils peuvent apprendre à le comprendre.

Il est possible de faire remarquer à un apprenant que la négation est réalisée sans ne dans c'est pas.

L'utilisation du déictique là associé à le bureau est également intéressante. La forme le... là relève ici plutôt d'une variation diatopique. 
D'autres éléments sont également intéressants à analyser avec des apprenants, tels que le tour de l'étudiante ligne 5 qui prend la forme d'une question suspensive. Cette forme est liée aux éléments de la situation et au genre de discours dans la mesure où produire un tel énoncé semble moins directif qu'un énoncé qui serait achevé avec un complément du type «le faire ». Ainsi l'énoncé «vous pouvez pas du tout le faire» parait plus intrusif et plus menaçant pour la face de l'interlocutrice à qui on demande explicitement de réaliser une action, ainsi que pour la face de la locutrice qui se trouve dans une position de formuler une demande probablement trop directe. Nous rencontrons d'autres exemples de ce type de questions dans le corpus FLEURON. L'exemple suivant illustre également l'adoucissement de la question grâce à son inachèvement syntaxique.

Extrait de la transcription de la vidéo « Inscription complète Erasmus 3 » dans FLEURON. L'agent administratif explique à une étudiante que des informations lui seront envoyées sur son adresse mail de l'université.

A : moi j'envoie les informations sur cette adresse d'accord

E : d'accord

A : donc il faudra bien regarder cette adresse quand je vous demande de passer au bureau pour avoir des renseignements hein euh c'est important je j'écrirai sur cette adresse l'uni- l'adresse de l'université Nancy 2

$E$ : et c'est moi qui dois euh le créer ou::

$\mathrm{A}:$ non elle se fera automatiquement hein quand vous ferez la connexion à internet

E : d'accord bien

L'étudiante demande si elle devra créer elle-même l'adresse en produisant une question alternative dont la deuxième partie n'est pas réalisée. Un enseignant qui analyse cette interaction avec un apprenant pourra pointer, dans la séquentialité de l'échange, cette pratique interactionnelle. Dans cet exemple, l'étudiante formule une question qui consiste à proposer, prioritairement, qu'elle réalise la tâche elle-même. Le statut d'étudiant semble influencer cette proposition. Il pourrait paraitre inapproprié de demander si une autre personne ou un service administratif pouvait se charger de cette tâche, avant même de proposer de la réaliser soi-même. En outre, cette façon de poser une question peut être enseignée comme une stratégie. Il n'est pas indispensable de connaitre l'alternative, ou les alternatives, pour interroger son interlocuteur. Dans l'exemple ci-dessus, on peut imaginer que l'étudiante ne sait pas quelle est la procédure ou qu'elle ne sait pas la verbaliser. La première partie de sa question alternative est suffisante pour que son interlocutrice comprenne la question est y réponde immédiatement, dans le tour suivant. Ces exemples montrent que les résultats des recherches sur les interactions verbales peuvent aider les enseignants à expliquer des phénomènes interactionnels inexistants dans les manuels ou grammaires traditionnelles. Ces résultats méritent d'être transposés à des fins didactiques pour des enseignants et des apprenants qui ne sont pas tous des analystes de la langue.

Pour conclure cette partie, nous allons présenter une dernière analyse réalisée conjointement par une enseignante et des apprenants. Cette analyse a été effectuée avec une interaction de FLEURON dont voici la capture d'écran comprenant l'image de la vidéo et la transcription. 


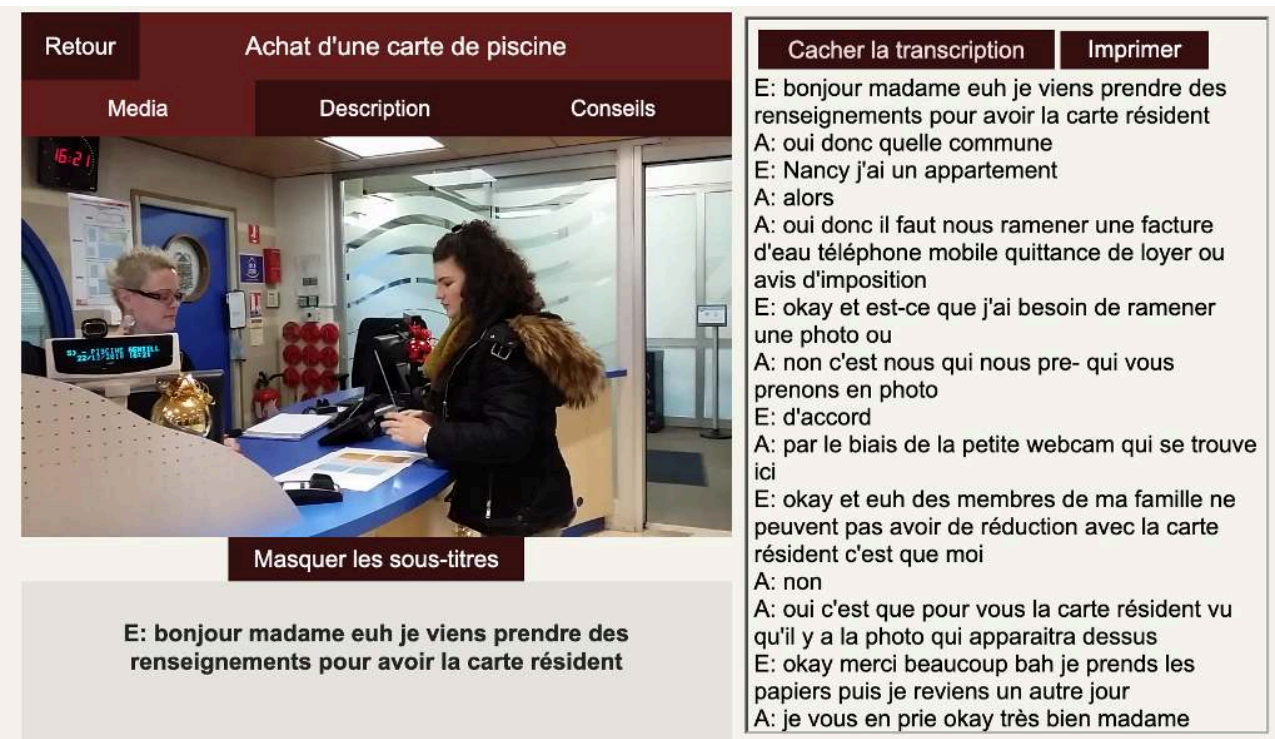

Figure 3 : Interaction dans la catégorie « Vie en dehors du campus » de FLEURON

La démarche proposée n'est pas exclusive. Elle peut s'articuler avec des activités de
compréhension orale plus classique, permettant d'évaluer la compréhension globale ou
détaillée de l'interaction. Cette démarche permet d'exposer les apprenants à la réalité
et à la variation des interactions et surtout elle permet aux apprenants de comprendre
des éléments qui ne sont habituellement pas enseignés en FLE. Les spécificités du
français parlé en interaction posent fréquemment des problèmes de compréhension
pour des apprenants, même pour les plus avancés. Cette démarche peut être complétée

La démarche proposée n'est pas exclusive. Elle peut s'articuler avec des activités de
compréhension orale plus classique, permettant d'évaluer la compréhension globale ou
détaillée de l'interaction. Cette démarche permet d'exposer les apprenants à la réalité
et à la variation des interactions et surtout elle permet aux apprenants de comprendre
des éléments qui ne sont habituellement pas enseignés en FLE. Les spécificités du
français parlé en interaction posent fréquemment des problèmes de compréhension
pour des apprenants, même pour les plus avancés. Cette démarche peut être complétée

La démarche proposée n'est pas exclusive. Elle peut s'articuler avec des activités de
compréhension orale plus classique, permettant d'évaluer la compréhension globale ou
détaillée de l'interaction. Cette démarche permet d'exposer les apprenants à la réalité
et à la variation des interactions et surtout elle permet aux apprenants de comprendre
des éléments qui ne sont habituellement pas enseignés en FLE. Les spécificités du
français parlé en interaction posent fréquemment des problèmes de compréhension
pour des apprenants, même pour les plus avancés. Cette démarche peut être complétée

La démarche proposée n'est pas exclusive. Elle peut s'articuler avec des activités de
compréhension orale plus classique, permettant d'évaluer la compréhension globale ou
détaillée de l'interaction. Cette démarche permet d'exposer les apprenants à la réalité
et à la variation des interactions et surtout elle permet aux apprenants de comprendre
des éléments qui ne sont habituellement pas enseignés en FLE. Les spécificités du
français parlé en interaction posent fréquemment des problèmes de compréhension
pour des apprenants, même pour les plus avancés. Cette démarche peut être complétée

La démarche proposée n'est pas exclusive. Elle peut s'articuler avec des activités de
compréhension orale plus classique, permettant d'évaluer la compréhension globale ou
détaillée de l'interaction. Cette démarche permet d'exposer les apprenants à la réalité
et à la variation des interactions et surtout elle permet aux apprenants de comprendre
des éléments qui ne sont habituellement pas enseignés en FLE. Les spécificités du
français parlé en interaction posent fréquemment des problèmes de compréhension
pour des apprenants, même pour les plus avancés. Cette démarche peut être complétée

La démarche proposée n'est pas exclusive. Elle peut s'articuler avec des activités de
compréhension orale plus classique, permettant d'évaluer la compréhension globale ou
détaillée de l'interaction. Cette démarche permet d'exposer les apprenants à la réalité
et à la variation des interactions et surtout elle permet aux apprenants de comprendre
des éléments qui ne sont habituellement pas enseignés en FLE. Les spécificités du
français parlé en interaction posent fréquemment des problèmes de compréhension
pour des apprenants, même pour les plus avancés. Cette démarche peut être complétée

une demande de renseignement ;

une assertion pour demander quelque chose: «je viens prendre des renseignements ";

le peu de politesse dans cet échange de service ne nuit pas à l'interaction (par exemple, l'agent (A) ne répond pas au bonjour de l'étudiante (E)) ;

et des éléments qui ne sont jamais abordés en formation linguistique :

une question alternative inachevée en « ou »;

un retour sur l'axe syntagmatique «c'est nous qui nous pre- qui vous prenons en photo ";

des petits mots pour commencer un tour de parole « alors oui donc » ou « non oui ».

Ces éléments ont naturellement attiré l'attention des apprenants. Là encore, certains de ces éléments ne sont pas toujours évidents à expliquer pour un enseignant de FLE. L'analyse sociolinguistique des interactions verbales se révèle utile si elle est adaptée à des besoins didactiques. En outre, des éléments administratifs importants ont été abordés puisqu'il est question dans cette vidéo de documents qui permettent d'attester d'un domicile. Ces éléments font partie du quotidien des apprenants qui effectuent de nombreuses démarches administratives (recherche d'un logement, ouverture d'une ligne téléphonique, abonnement de transport, etc.). 
par une recherche d'un item dans le concordancier de FLEURON afin d'observer davantage de données et de saisir les règles de fonctionnement de l'item.

\subsection{Analyse distributionnelle avec un concordancier}

Certaines analyses des interactions verbales peuvent bénéficier d'un outil d'aide au repérage des phénomènes identifiés préalablement. C'est le cas par exemple de l'analyse présentée précédemment du marqueur genre. Pour réaliser cette analyse, le corpus TCOF a été interrogé par un concordancier, outil de la linguistique de corpus, afin d'extraire la totalité des occurrences de genre. Cette méthodologie d'exploration des corpus peut être transposée en didactique, c'est ce que Johns (1991) a appelé le data-driven learning, traduit en français par apprentissage sur corpus (ASC) (Boulton \&Tyne 2014). Dans les travaux anglo-saxons, cette approche est née de la volonté de trouver des applications à l'exploitation linguistique des corpus dans le processus d'enseignement et d'apprentissage des langues. En d'autres termes, cette approche vise à mettre un concordancier au service de l'enseignement et de l'apprentissage de la langue. Elle a donné naissance à de nombreuses expériences et travaux scientifiques mais rarement concernant l'exploitation des corpus oraux, à part quelques exceptions (voir par exemple Zorzi 2001; Mauranen 2004 pour l'anglais ou Kerr 2013 pour le français), et encore plus rarement des corpus multimodaux (Braun 2007). À notre connaissance, excepté dans le cadre du projet Sacodeyl ${ }^{11}$ destiné à des adolescents, cette approche n'a jamais été expérimentée en France avec des corpus d'interactions pour apprendre à interagir en français.

Dans cette partie, l'objectif n'est pas de présenter les travaux déjà réalisés dans le domaine de l'ASC. De nombreuses méta-analyses ont été effectuées, voir notamment Chambers (2010) ; Cobb \& Boulton (2015) ; Boulton (2017) ou encore Boulton \& Cobb (2017). Notre objectif est de montrer de quelle façon les apprenants peuvent se servir $\mathrm{du}$ concordancier de FLEURON pour accroitre leurs compétences sociointeractionnelles à l'oral en français. Ce concordancier permet d'effectuer des recherches de chaines de caractères dans toutes les transcriptions du corpus (André \& Ciekanski 2018). Il affiche les résultats sous la forme de ligne de concordances. Toutefois, la particularité de ce concordancier multimodal réside dans son alignement texte/son/vidéo. Il suffit de cliquer sur une occurrence de la liste pour que la ressource de laquelle est extraite cette occurrence apparaisse et que la lecture soit positionnée au moment où l'occurrence est prononcée. La figure ci-dessous illustre les fonctionnalités du concordancier avec la recherche du marqueur genre. 


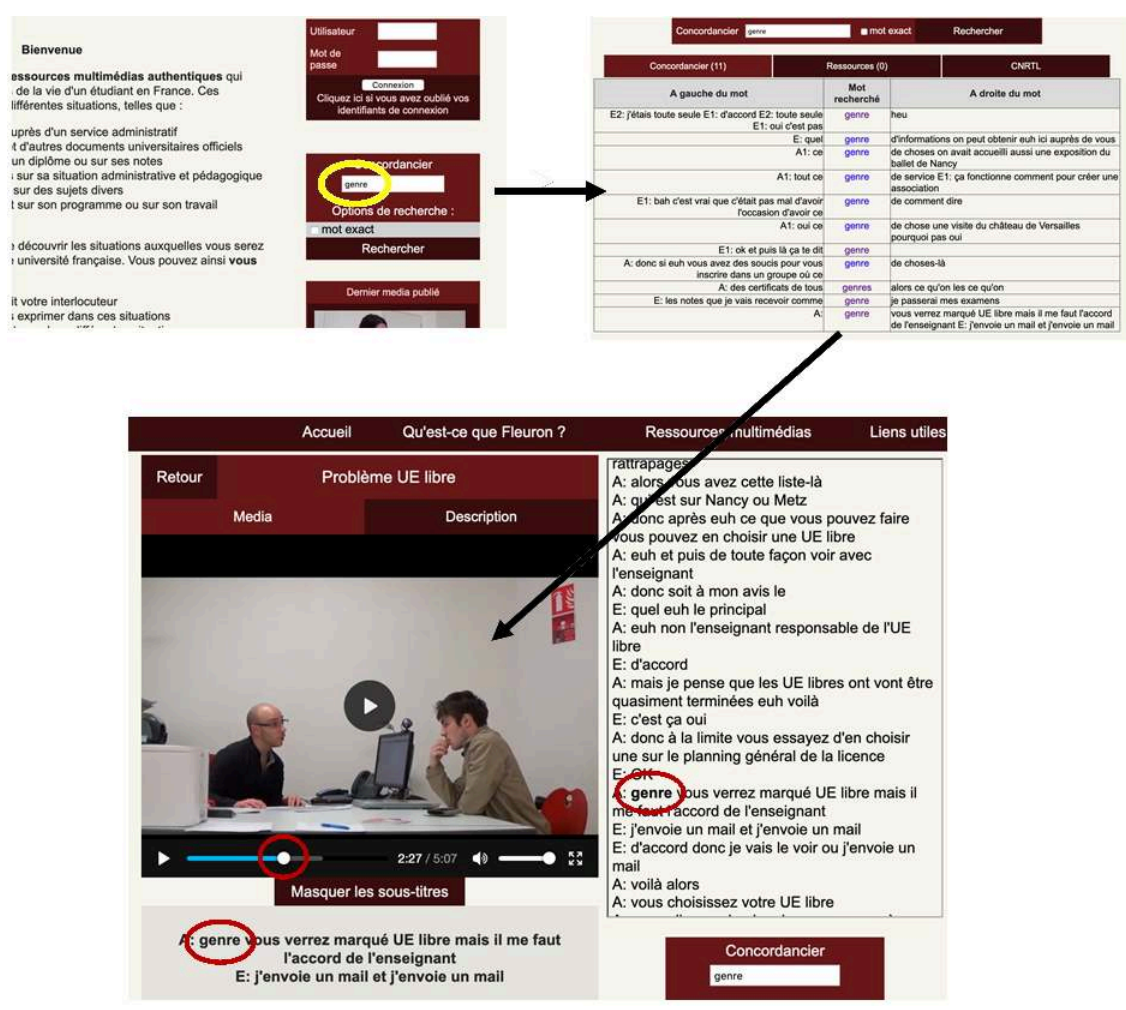

Figure 4 : Fonctionnement du concordancier multimodal de FLEURON concordancier qui se trouve sur la page d'accueil de FLEURON (image en haut à gauche) ${ }^{12}$, le résultat est affiché en liste (image en haut à droite) avec le cotexte gauche et droite. Si un apprenant souhaite écouter une occurrence ou obtenir plus de cotexte que celui qui est affiché dans la liste, il peut cliquer sur cette occurrence et la ressource apparait (image du bas). Celle-ci est calée sur l'occurrence recherchée qui apparait en gras dans la transcription. Si l'apprenant souhaite naviguer dans la vidéo, il peut cliquer où il le souhaite dans la transcription pour visualiser la partie souhaitée. Par exemple, dans la figure ci-dessus, l'apprenant peut cliquer sur "d'accord " prononcé par E (5 tours avant genre) et la vidéo se positionnera sur ce segment. prometteuses. Elles ont été effectuées avec des apprenants de différents niveaux (de débutants à avancés, de peu scolarisés à très diplômés), avec différents types d'accompagnement (guidage fort et l'accompagnement léger après une présentation du dispositif) et dans différentes structures (université, organismes de formation, associations). Les apprenants observent les données, créent des catégories et induisent les règles de fonctionnement des items recherchés selon la méthodologie du DDL (Johns 1991). Pour illustrer cette méthodologie, nous proposons d'examiner les démarches entreprises par des apprenants. Ces derniers font de la sociolinguistique de corpus sans le savoir, c'est-à-dire qu'ils réalisent des analyses distributionnelles des mots qu'ils recherchent dans le concordancier en observant les contextes ainsi que les cotextes droits et gauches (André 2018). Par exemple, lors de l'analyse conjointe de l'interaction «Paiement en espèce » (Figure 2), des apprenants ont souhaité poursuivre l'analyse de «je voulais» dans l'énoncé «alors moi j'étais venue pour une demande toute simple hum en fait euh c'est pour mon inscription voilà je voulais payer en espèces ». Nous les 
avons donc orientés vers la recherche de " voulais» dans le concordancier. Les résultats sont présentés dans la figure 5 .

\begin{tabular}{|c|c|c|}
\hline A gauche du mot & $\begin{array}{l}\text { Mot } \\
\text { recherché }\end{array}$ & A droite du mot \\
\hline A : euh je & voulais & $\begin{array}{l}\text { juste vous demander vous suivez les cours de } \\
\text { français intensif }\end{array}$ \\
\hline$E: j e$ & voulais & payer en espèces \\
\hline $\begin{array}{r}\text { E: je voudrais découvrir un petit peu la France A: oui } \\
E: \text { et je }\end{array}$ & voulais & savoir ce que vous pouvez me proposer pour euh \\
\hline$E: j e$ & voulais & être sûre \\
\hline E: c'est tout ce que je & voulais & savoir A: ouais $E$ : je vous remercie \\
\hline E1 : c'est pour ça je & voulais & $\begin{array}{l}\text { savoir A : ils vont être bons hein on peut pas savoir } \\
\text { enfin }\end{array}$ \\
\hline A : c'est vrai que euh je ne & voulais & $\begin{array}{l}\text { pas vous donner trop de documents E : oui A : au } \\
\text { début }\end{array}$ \\
\hline A : alors je & voulais & vous demander \\
\hline A : alors ce que je & voulais & $\begin{array}{l}\text { savoir est-ce que vous avez eu l'occasion de } \\
\text { regarder un petit peu les }\end{array}$ \\
\hline A1: voilà donc je & voulais & juste passer le relais à Sylvie \\
\hline A: voilà ce que je & voulais & $\begin{array}{l}\text { dire pour heu pour vous décrire un petit peu le le } \\
\text { CROUS }\end{array}$ \\
\hline A: non mais c'est pas E: d'ailleurs c'est ça que je & voulais & $\begin{array}{l}\text { vous demander faut que je ramène quoi pour } \\
\text { l'attestation de }\end{array}$ \\
\hline
\end{tabular}

Figure 5 : Résultats de la recherche de « voulais » dans le concordancier de FLEURON.

52 Le corpus FLEURON compte seulement douze occurrences de voulais ${ }^{13}$. Les apprenants ont ainsi pu analyser toutes les occurrences. Voici, pas à pas, la démarche qu'un étudiant a effectuée, avec une intervention minimale de l'enseignant (essentiellement des interrogations du type : qu'est-ce que ça signifie ici accompagnées d'un pointage sur l'écran pour inciter l'apprenant à s'interroger sur les données) :

Il a d'abord observé toutes les occurrences les unes après les autres, dans leur ordre d'apparition à l'écran.

Il a commencé par faire une catégorie avec les occurrences qui étaient associées à une demande (en commençant par mettre dans cette catégorie les occurrences se trouvant dans des énoncés ou le mot demande était présent). Les occurrences 1, 2, 3 , $5,6,8,9$ et 12 ont été classées dans cette catégorie.

Il a eu recours à la vidéo (contexte plus large) pour comprendre l'occurrence 4. Le cotexte de la ligne de concordance est le suivant : euh je voudrais savoir euh est-ce que euh j'ai vraiment validé mes deux années euh je voulais être sûre. Avec la cooccurrence de je voudrais savoir, l'étudiant a ajouté l'occurrence 4 dans la première catégorie (avec une demande).

En allant visionner les 3 occurrences restantes, il s'est rendu compte que les 10 et 11 étaient prononcées lors de conférences d'accueil des étudiants étrangers. Pour ces deux occurrences, le locuteur utilise voulais alors qu'il est sur le point de faire quelque chose (passer le relai, dire quelque chose).

L'occurrence 7 est la seule occurrence de voulais qui a le sens que l'apprenant connait, un synonyme de souhaitais, et qui exprime un événement passé (la locutrice fait référence à un rendez-vous précédent).

La démarche présentée suit celle en trois phases préconisée par l'ASC : observation des données, catégorisation et généralisation (Johns 1991). Rapidement, les apprenants ont compris que le passé du verbe vouloir pouvait exprimer autre chose que des faits passés. Il permet d'exprimer une action future, comme nous l'avons déjà expliqué lors de l'analyse de l'interaction de la ressource «Paiement en espèces » dont l'occurrence correspond à la seconde occurrence de la liste ci-dessus. Les interventions collectives des apprenants sont reproduites en substance ci-dessous :

ça sert à faire une formule de politesse,

ça sert dans des situations administratives,

avec les locuteurs qui ne se connaissent pas, 
ça sert à préparer une question comme dans " je voulais vous demander »,

c'est avant une question pour être plus poli,

ça sert à dire qu'on est sur le point de faire quelque chose qu'on se prépare à faire

quelque chose.

C'est en analysant chacune des occurrences de voulais que les apprenants ont réussi à comprendre ses usages et son fonctionnement. Dans le cadre de cette étude, nous n'examinons pas les effets à long terme de l'apprentissage sur corpus. Néanmoins, à l'issue de cet exercice, certains apprenants ont eux-mêmes créé des activités de systématisation (Carette 2001) et surtout de mises en pratique en utilisant, pendant la séance de formation, des énoncés appropriés comprenant voulais. De plus, certains des apprenants ont souligné qu'à partir de cette séance, non seulement ils comprenaient voulais mais qu'ils seraient désormais également capables de l'utiliser.

Par ailleurs, l'analyse sociolinguistique des interactions verbales peut également être utile pour saisir des fréquences d'utilisations en fonction d'éléments de la situation de communication. Comme nous l'avons précisé dans la première partie, le marqueur genre est essentiellement utilisé par des locuteurs assez jeunes (entre 15 et 25 ans) engagés dans des conversations informelles. La question de l'utilisation et du sens de genre en interaction revient très fréquemment en classe de FLE. Cette question désarçonne généralement les enseignants qui ne sont pas des analystes de la langue et qui se trouvent souvent démunis pour répondre aux interrogations de leurs apprenants. C'est pourquoi, dans un premier temps, il est important de transposer ou de donner un accès aux résultats des analyses interactionnelles à des enseignants voire à des apprenants. C'est ce que proposent par exemple les projets CLAPI-FLE (mentionné précédemment) et CORAIL (Corpus ORaux pour les Apprenants à partir des études en Interaction et en Linguistique de l'oral, piloté par Carole Etienne à ICAR) qui cherche à fournir, à des apprenants, un accès direct à des interactions verbales authentiques expliquées et documentées à des fins didactiques. Au-delà de ces ressources pour les enseignants et les apprenants de FLE, l'utilisation directe d'un concordancier peut être mise au service d'une analyse de la variation et de la fréquence d'apparition de certains phénomènes de l'oral et ainsi répondre aux interrogations des apprenants et des enseignants. Lors de différentes expérimentations du concordancier de FLEURON, des apprenants ont souhaité chercher des items tels que: cependant, toutefois, par conséquent, en outre, car, ainsi ou encore genre. Les six premiers items (tous sauf genre) ont d'abord été recherchés dans FLEURON avec les résultats suivants :

\begin{tabular}{|l|l|}
\hline Item recherché & Nombre d'occurrence(s) dans FLEURON \\
\hline cependant & 0 \\
\hline toutefois & 1 \\
\hline par conséquent & 0 \\
\hline en outre & 0 \\
\hline car & 0 \\
\hline
\end{tabular}


ainsi 8

Tableau 1 : Nombre d'occurrences de six items dans FLEURON. corpus écrits, notamment dans Frantext ${ }^{14}$, dans sa partie contemporaine, et dans le corpus du journal Est Républicain, accessible via la plateforme ScienQuest ${ }^{15}$. Les résultats sont extrêmement conséquents, même si les corpus ne sont pas comparables en taille. Par exemple, en sélectionnant, dans la partie « démonstration » de Frantext (c'est-à-dire celle qui est accessible librement et gratuitement), uniquement les quatre textes du XXème siècle, nous trouvons 142 occurrences de cependant. Dans le journal Est Républicain, nous trouvons 459 occurrences pour les vingt exemplaires de février 2003. Ce simple constat quantitatif peut orienter les objectifs d'apprentissage. Par exemple, lorsque l'on souhaite enseigner ou apprendre à interagir à l'oral en français, il n'est sans doute pas utile d'apprendre prioritairement à comprendre et/ou à produire cependant.

La recherche d'occurrences avec un concordancier peut donner d'autres informations que celles qui concernent des usages différents à l'oral et à l'écrit. Par exemple, ce marqueur genre, souvent questionné par les apprenants, n'apparait pas dans tous les genres de discours et n'est pas produit par tous les types de locuteurs. Les deux images suivantes sont des extraits des recherches de genre respectivement dans les corpus TCOF et FLEURON.

\begin{tabular}{|c|c|c|c|c|c|}
\hline$\hat{\varnothing}$ & RegExp & Min/Maj & $\begin{array}{l}\text { Np gccurren } \\
\text { Nb fichiers: }\end{array}$ & $\begin{array}{l}\text { ces: } 666 \\
125 / 253 \\
\text { s : } 30 / 128\end{array}$ & \\
\hline Num & Fichier & \multicolumn{2}{|c|}{ Contexte Gauche } & Occurrence & Contexte Droit \\
\hline 541 & Afev_KEM_12 & \multicolumn{2}{|c|}{ fait un dessin pour faire } & genre & fait fait un pouce comme \\
\hline 542 & Afev_KEM_12 & \multicolumn{2}{|c|}{ c'est pas beaucoup hein } & genre & un petit truc comme ça \\
\hline 543 & Afev_KEM_12 & \multicolumn{2}{|c|}{ niveau de facebook un groupe } & genre & AFEV où on peut partager \\
\hline 544 & CE_Pooky_QUE... & \multicolumn{2}{|c|}{ de certaines machines outils } & genre & piler buron et puis du \\
\hline 545 & Corpus_LAS_12 & \multicolumn{2}{|c|}{ comment la > je coupe } & genre & déjà un truc en trois \\
\hline 546 & Corpus_LAS_12 & \multicolumn{2}{|c|}{ suis obligée de me lever } & genre & vers sept heures et quart \\
\hline 547 & Corpus_LAS_12 & \multicolumn{2}{|r|}{ en $>$ sais rien moi } & genre & en plonge là avec les \\
\hline 548 & Corpus_LAS_12 & \multicolumn{2}{|r|}{ à la fac on était } & genre & en cours de maths et \\
\hline 549 & Corpus_LAS_12 & \multicolumn{2}{|r|}{ tu sais puis il était } & genre & huit heures et quart puis \\
\hline 550 & Corpus_LAS_12 & \multicolumn{2}{|c|}{ amphi il devait y avoir } & genre & vingt personnes tu sais \\
\hline 551 & Corpus_LAS_12 & \multicolumn{2}{|c|}{ du coup maintenant euh mais } & genre & tu es à côté > \\
\hline 552 & Corpus_LAS_12 & \multicolumn{2}{|c|}{ je voudrais bien tester mais } & genre & tu c'est trop bon \\
\hline 553 & Corpus_LAS_12 & \multicolumn{2}{|c|}{ trop bon quoi enfin mais } & genre & c'est c'est pas \\
\hline 554 & Experiences_Y... & \multicolumn{2}{|r|}{ les achètes c'est un } & genre & de congés sans solde que \\
\hline 555 & Foot_MAR_12 & \multicolumn{2}{|c|}{ trucs euh pas banals mais } & genre & aller dans les écoles faire \\
\hline 556 & Foot_MAR_12 & \multicolumn{2}{|r|}{ à Paris on a voulu } & genre & trois quatre après euh voilà \\
\hline 557 & Fumuj_HEN_12 & \multicolumn{2}{|c|}{ euh enfin de proposer ce } & genre & de de création quoi et \\
\hline 558 & Fumui HFN 12 & \multicolumn{2}{|c|}{ moi c'est vraiment le } & aenre & de truc et encore auiourd' \\
\hline
\end{tabular}

Figure 6 : Extraits des résultats des recherches de genre dans TCOF 


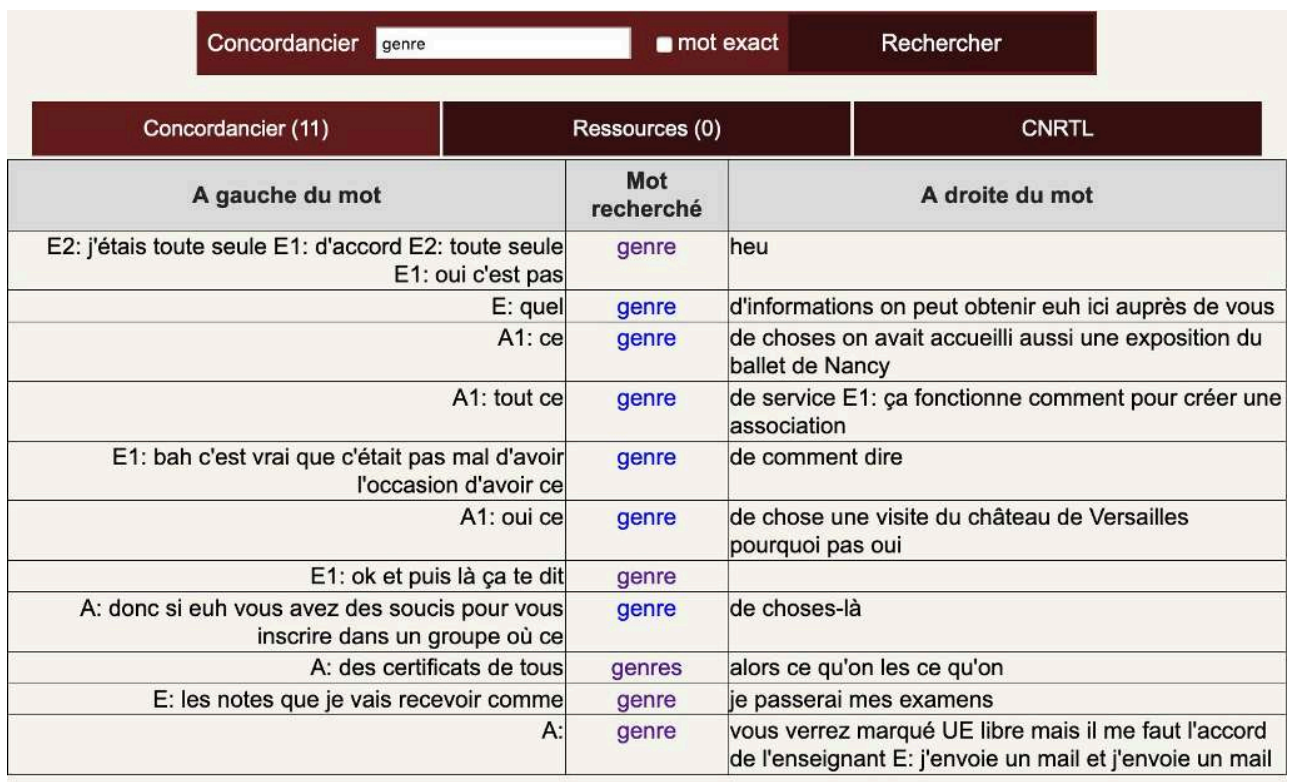

Figure 7 : Extraits des résultats des recherches de genre dans FLEURON

Dans le corpus FLEURON, nous trouvons seulement 11 occurrences du marqueur genre. En outre, seulement quatre occurrences fonctionnent comme marqueur. Les autres genre sont inclus dans des expressions telles que ce genre de (chose), tous genres ou quel genre. Dans le corpus TCOF qui compte de nombreuses conversations entre des locuteurs qui se connaissent présentent 666 occurrences de genre. Toutes les interactions du corpus ne comptent pas de genre, seules 125 fichiers sur les 253 interrogés comporte au moins une occurrence et tous les locuteurs ne produisent pas genre, seuls 30 locuteurs en produisent sur 128 . Ces résultats peuvent être exploités par des enseignants et des apprenants de FLE. Il n'est pas utile de faire une analyse très détaillée des résultats mais il est intéressant de croiser ces derniers avec les métadonnées des corpus, c'est-à-dire avec les éléments situationnels. Ainsi, une première déduction peut être faite concernant les situations formelles de FLEURON (essentiellement des interactions dans des services administratifs): elles sont peu propices à l'apparition de genre. De plus, en cliquant sur les 4 occurrences de genre en tant que marqueur, nous pouvons constater dans quelles circonstances apparaissent ces occurrences. Le tableau 2 ci-dessous mentionne les éléments situationnels.

\begin{tabular}{|l|l|}
\hline Occurrences & Situation de communication et genre de discours \\
\hline E2: j'étais toute seule & \\
E1: d'accord & Une étudiante étrangère (E2) raconte à une autre étudiante (E1) \\
E2: toute seule & comment elle a vécu la visite médicale. C'est une conversation \\
E1: oui c'est pas genre heu & informelle entre deux étudiantes de la même promotion. \\
E2: parce que le rendez-vous \\
est $* *$ il y avait des autres \\
personnes
\end{tabular}




\begin{tabular}{|c|c|}
\hline $\begin{array}{l}\text { E1: ok et puis là ça te dit genre } \\
\text { trente premières gra- minutes } \\
\text { gratuites et puis si }\end{array}$ & \\
\hline $\begin{array}{l}\text { E2: c'est ça } \\
\text { E1: si tu l'as pas reposé au bout } \\
\text { d'une demi-heure } \\
\text { E2: c'est ça } \\
\text { E1: tu payes un euro en plus }\end{array}$ & $\begin{array}{l}\text { Une étudiante (E1) explique à une autre étudiante (E2) comment } \\
\text { louer un vélib'. L'explication se déroule dans la rue, face à une } \\
\text { borne de location. Les deux étudiantes sont des amies. }\end{array}$ \\
\hline $\begin{array}{l}\text { E: euh j'aurai comment } \\
\text { comment pour savoir les notes } \\
\text { je les trouve en ligne aussi } \\
\text { A: les notes } \\
\text { E: les notes que je vais recevoir } \\
\text { comme genre je passerai mes } \\
\text { examens } \\
\text { A: bien sûr c'est sur votre ENT }\end{array}$ & $\begin{array}{l}\text { Un étudiant (E) se renseigne auprès de la gestionnaire de sa } \\
\text { licence (A) pour savoir comment il pourra accéder à ses notes une } \\
\text { fois qu'il aura passé ses examens. Cet échange a lieu pendant une } \\
\text { permanence du service scolarité. }\end{array}$ \\
\hline $\begin{array}{l}\text { A: donc à la limite vous essayez } \\
\text { d'en choisir une sur le planning } \\
\text { général de la licence } \\
\text { E: OK } \\
\text { A: genre vous verrez marqué } \\
\text { UE libre mais il me faut l'accord } \\
\text { de l'enseignant } \\
\text { E: j'envoie un mail et j'envoie } \\
\text { un mail d'accord donc je vais le } \\
\text { voir ou j'envoie un mail }\end{array}$ & $\begin{array}{l}\text { Un personnel administratif jeune (A) explique le fonctionnement } \\
\text { des Unités d'Enseignement (UE) libres à un étudiant (E) lors d'une } \\
\text { permanence du service scolarité. }\end{array}$ \\
\hline
\end{tabular}

Tableau 2 : Les occurrences du marqueur genre dans FLEURON et les éléments situationnels

59 Ainsi, il existe différentes façons d'exploiter des corpus à des fins didactiques. La recherche d'occurrences dans le concordancier ou l'ASC n'est pas non plus une méthodologie exclusive, elle peut être associée à l'analyse des interactions que nous avons présentée précédemment ainsi qu'à d'autres méthodologies et à l'utilisation d'autre ressources (CLAPI-FLE par exemple). Lors des multiples expérimentations que nous avons pu faire de cette approche, nous avons constaté que les apprenants réussissent à induire les règles de fonctionnement de la langue, avec plus ou moins d'aide de la part de l'enseignant. D'autres études, sur l'apprentissage de l'anglais écrit, montrent que les éléments saisis de cette façon sont mieux retenus donc plus facilement acquis que des recherches plus traditionnelles, avec un dictionnaire par exemple (Landure 2011). Les expérimentations de FLEURON sont encourageantes parce qu'elles montrent que la méthodologie de l'ASC peut être déployée avec des corpus oraux et multimodaux. Des études longitudinales sont désormais à organiser afin d'examiner si cette méthodologie est réellement efficace sur le long terme. 


\section{Conclusion}

Nous avons cherché à montrer que les liens entre sociolinguistique et didactique méritent d'être resserrés voire, parfois, d'être créés afin d'améliorer les formations en FLE en ce qui concerne les interactions à l'oral, et d'aider les enseignants et les apprenants à comprendre le fonctionnement du français parlé en interaction. Nous souhaitons insister sur la nécessité de mettre les résultats de la recherche en sociolinguistique des interactions verbales à la disposition des enseignants et des apprenants de langue. Ces derniers ne sont pas des spécialistes de la langue mais il est légitime qu'ils puissent s'approprier les connaissances qui leur sont utiles, comme celles concernant certaines formes questions et les apparitions du marqueur genre, dont les analyses sont présentées dans article. Cette mise à disposition n'est pas toujours évidente, elle demande un travail de transposition à co-construire entre les chercheurs et les praticiens. Cette collaboration est une des conditions de réussite de l'exploitation des corpus à des fins didactiques. Dans cette perspective, les enseignants de FLE doivent également être formés à l'utilisation de différentes ressources : corpus d'interactions, outils d'interrogation des données et didactisation des résultats des recherches sur les interactions. Ils peuvent ainsi aider les apprenants à entrer dans les démarches et les activités cognitives proposées. Les apprenants doivent également être formés, ils ne sont plus simplement passifs, ils sont acteurs de leur apprentissage, en analysant les interactions verbales ou en analysant les résultats de l'interrogation des corpus. Il existe ainsi différentes façons d'exploiter la sociolinguistique des interactions verbales à des fins didactiques, elles ne sont pas exclusives mais complémentaires pour l'amélioration des compétences socio-interactionnelles des apprenants.

\section{BIBLIOGRAPHIE}

ALBERDI, C., ÉTIENNE, C., JOUIN-CHARDON, É., 2018, « Les apports des corpus d'interactions naturelles en situation de classe : enjeux et pratique ", Action Didactique 1, p. 55-70. En ligne : http://univ-bejaia.dz/action-didactique/ad1.html.

ANDRÉ, V., 2019, Sociolinguistique des interactions verbales et exploitations didactique, Habilitation à diriger des recherches, Lyon : Université Lumière Lyon 2. En ligne : https://perso.atilf.fr/ VirginieAndre/cv/.

ANDRÉ, V., 2005, « Oui non : une pratique discursive sous influence », Marges Linguistiques 9, p. 195-213. En ligne : http://www.revue-texto.net/Parutions/Marges/Marges_sommaire.html.

ANDRÉ, V., 2010, « Éléments de construction collaborative du discours au sein de réunions de travail : la reprise et le couple oui non », Pratiques 147/148, p. 199-222.

ANDRÉ, V., 2016, « FLEURON : Français Langue Étrangère Universitaire - Ressources et Outils Numériques. Origine, démarches et perspectives ", Mélanges Crapel 37, p. 69-92. En ligne : http:// www.atilf.fr/IMG/pdf/art3.pdf. 
ANDRÉ, V., 2018, « Nouvelles actions didactiques : faire de la sociolinguistique de corpus pour enseigner et apprendre à interagir en français langue étrangère ", Action didactique 1, p. 71-88. En ligne : http://univ-bejaia.dz/pdf/ad1/Andre.pdf.

ANDRÉ, V., CIEKANSKI, M., 2018, « Apprendre à interagir à l'oral à partir d'un concordancier multimodal : effets sur le développement de la conscience langagière et sur l'autonomie de l'apprenant dans le dispositif FLEURON », dans C. Dejean, F. Mangenot, E. Nissen, T. Soubrié (coord.), EPAL - Échanger Pour Apprendre en Ligne, Juin 2018, Grenoble, France.

AUSTIN, J. L., 1962 (trad. française 1970), Quand dire c'est faire, Paris : Seuil.

BÉGUELIN, M.-J., COVENEY, A., GURYEV, A. (dir.), 2018, L'interrogative en français, Bern : Peter Lang.

BOULTON, A., 2017, « Corpora in language teaching and learning », Language Teaching, 50(4), p. 483-506.

BOULTON, A., COBB, T., 2017, « Corpus use in language learning: a meta-analysis », Language Teaching 67, p. 348-393.

BOULTON, A., TYNE, H., 2014, Des Documents Authentiques aux Corpus. Démarches pour l'Apprentissage des Langues. Paris : Didier.

BOUTET, J., 1994, Construire le sens. Bern : Peter Lang.

BRAUN, S., 2007, « Integrating corpus work into secondary education: From data-driven learning to needs-driven corpora ", ReCALL 19(3), p. 307-328.

CARETTE, E., 2001, « Mieux apprendre à comprendre l'oral en langue étrangère », Le français dans le monde. Recherches et applications. Numéro spécial : Oral : variabilité et apprentissage, p. 126-142.

CHAMBERS, A., 2010, « What is data-driven learning? », dans A. O'Keeffe, M. McCarthy (ed.), The Routledge Handbook of Corpus Linguistics, New York: Routledge, p. 345-358.

CHAUVEAU-THOUMELIN, P., 2016, « De l'exemplification à la catégorisation approximative : étude de la construction [[X] SN genre [Y] SN] », SHS Web of Conferences Vol. 27, p. 12005, EDP Sciences.

COBB, T., BOULTON, A., 2015, « Classroom applications of corpus analysis », dans D. Biber, R. Reppen (eds.), The Cambridge handbook of English corpus linguistics, Cambridge: Cambridge University Press, p. 478-497.

COVENEY, A., 1996, Variability in spoken French: A sociolinguistic study of interrogation and negation, Exeter: Elm Bank Publications.

DEBAISIEUX, J.-M., BOULTON, A., 2007, « Alors la question c'est...? Questions pragmatiques et annotation pédagogique des corpus », Cahiers de l'AFLS 13(2), p. 31-59.

DETEY, S., DURAND, J., LAKS, B., LYCHE, C. (éds.), 2010, Les variétés du français parlé dans l'espace francophone: ressources pour l'enseignement, Paris : Ophrys.

DETEY, S., LYCHE, C., TCHOBANOV, A., DURAND, J., LAKS, B., 2009, « Ressources phonologiques au service de la didactique de l'oral : le projet PFC-EF », dans Mélanges CRAPEL 31, p. 223-236.

DOSTIE, G., PUSCH, C. D., 2007, « Présentation. Les marqueurs discursifs. Sens et variation », Langue française 154, p. 3-12.

FLEISCHMAN, S., 1998, « Des jumeaux du discours : genre et like », La linguistique 34/2, p. 31-47.

GIROUD, A., SURCOUF, C., 2016, « De "Pierre, combien de membres avez-vous ?" à "Nous nous appelons Marc et Christian" : réflexions autour de l'authenticité dans les documents oraux des 
manuels de FLE pour débutants », SHS Web of Conferences 27. CMLF 2016. En ligne : https://doi.org/ 10.1051/shsconf/20162707017.

GOFFMAN, E., 1974, Les rites d'interaction, Paris : Les Éditions de Minuit.

GOLOPENTIA, S., 1988, «Interaction et histoire conversationnelle », dans J. Cosnier, N. Gelas, C. Kerbrat-Orecchioni (dir.), Échanges sur la conversation, Paris : Éditions du CNRS, p. 69-81.

HYMES, D., 1972, « Models of the interaction of language and social life », dans J. Gumperz, D. Hymes (eds), Directions in Sociolinguistics. The Ethnography of Communication, Oxford: Basil Blackwell, p. 35-71.

JOHNS, T., 1991, "Should you be persuaded: Two samples of data-driven learning materials ", dans T. Johns, P. King (dir.), Classroom Concordancing, English Language Research Jounral 4, p. 1-16.

KERBRAT-ORECCHIONI, C., 1991, La question, Lyon : Presses universitaires de Lyon.

KERBRAT-ORECCHIONI, C., 2001, Les actes de langage dans le discours, Paris : Nathan.

KERBRAT-ORECCHIONI, C., TRAVERSO, V., 2004, « Types d'interactions et genres de l'oral », Langages 153, p. 41-51.

KERR, B., 2013, « Grammatical description and classroom application. Theory and practice un data-driven learning ", Bulletin VALS-ASLA 97, p. 17-39.

LANDURE, C., 2011, « Data-Driven Learning : Apprendre et enseigner à contre-courant », Mélanges CRAPEL 32, p. 163-178.

MAURANEN, A., 2004, « Spoken corpus for ordinary Learner », dans J. Sinclair (ed.), How to Use Corpora in Langugage Teaching, Amsterdam: Johns Benjamins, p. 89-105.

MONDADA, L., 2006, « La pertinence du contexte : Contributions de l'ethnométhodologie et de l'analyse conversationnelle, Verbum tome XXVIII, p. 2-3.

MOREL, M. A., DANON-BOILEAU, L., 1998, Grammaire de l'intonation l'exemple du français, Paris : Editions Ophrys.

ROSIER, L., 2002, « Genre : le nuancier de sa grammaticalisation », Travaux de linguistique 1, p. $79-88$.

ROSSARI, C., BEAULIEU-MASSON, A., COJOCRIU, C., RAZGOULIAEVA, A. (dir.), 2005, Les états de la question, Montréal : Nota Bene.

SEARLE, J., 1969/Trad. française 1972, Les actes de langage, Paris : Hermann.

SURCOUF, C., AUSONI, A., 2018, Création d'un corpus de français parlé à des fins pédagogiques en FLE : la genèse du projet FLORALE, Études en didactique des langues 31, p. 71-91. En ligne : http:// www.lairdil.fr/revue-edl-issn-2258-1510-626.html.

TRAVERSO, V., 2016, Décrire le français parlé en interaction, Paris : Éditions Ophrys.

VLADIMIRSKA, E., 2016, « Entre le dire et le monde : le cas du marqueur discursif genre », dans H. Bat-Zeev Shyldkrot, S. Adler, M. Asnes (eds), Nouveaux regards sur l'approximation et la précision, Paris : Honoré Champion, p. 195-208.

YAGUELLO, M., 1998, Petits faits de langue, Paris : Éditions du Seuil.

ZORZI, D., 2001, « The pedagogic use of spoken corpora: Learning discourse markers in Italian », dans G. Aston (ed.), Learning with corpora, Houston: Athelstan, p. 85-107. 


\section{NOTES}

1. https://www.cnrtl.fr/corpus/tcof/

2. Accessible librement et gratuitement : https://fleuron.atilf.fr/

3. Traitement de corpus oraux en français : https://www.cnrtl.fr/corpus/tcof/. Les conventions de transcription sont accessibles sur le site.

4. Le corpus utilisé ici n'est que partiellement diffusé. Une centaine d'enregistrements, explorés dans cette étude, sont en cours de traitement (vérification et anonymisation).

5. Selon les conventions de TCOF, le premier locuteur qui prend la parole est identifié L1, le second $\mathrm{L} 2$, etc.

6. L'ESPE (École Supérieure du Professorat et de l'Éducation) est le nouveau nom de l'IUFM (Institut Universitaire de Formation des Maîtres). Depuis septembre 2019, l'ESPE est devenu l'INSPE (Institut National Supérieur du Professorat et de l'Éducation).

7. Cette étude fera l'objet d'une prochaine publication dans la revue Langages.

8. http://clapi.ish-lyon.cnrs.fr/FLE/.

9. https://florale.unil.ch/.

10. https://www.projet-pfc.net/le-projet-pfc-ef/.

11. https://www.um.es/sacodeyl/

12. Le concordancier est également accessible depuis toutes les pages du site.

13. Au moment de la rédaction de cet article.

14. https://www.frantext.fr/.

15. https://corpora.aiakide.net/scientext20/

\section{RÉSUMÉS}

Dans cet article, nous montrons comment les enseignants et les apprenants de Français Langue Étrangère (FLE) peuvent tirer profit des analyses sociolinguistiques des interactions verbales. Nous explorons différentes façons d'exploiter ces analyses à des fins didactiques, notamment en utilisant les outils de la linguistique de corpus. Nous illustrons ces nouvelles méthodologies didactiques avec des expérimentations réalisées avec le corpus multimédia du dispositif d'apprentissage en ligne FLEURON (https://fleuron.atilf.fr/). Nous présentons de quelles façons l'analyse des interactions dans leur temporalité ainsi que l'analyse de listes d'occurrences peuvent être investies en classe de langue par les enseignants ou en autonomie par les apprenants.

In this paper, we show how teachers and learners of French as a Foreign Language (FFL) can benefit from sociolinguistic analyses of verbal interactions. We explore different ways of using these analyses for didactic purposes, in particular by using corpus linguistics tools. We illustrate these new didactic methodologies with experiments carried out with the multimedia corpus of the FLEURON e-learning system (https://fleuron.atilf.fr/). We present how the analysis of interactions in their temporality as well as the analysis of lists of occurrences can be invested in language classes by teachers or in autonomy by learners. 
INDEX

Mots-clés : interactions verbales, corpus multimodal, Apprentissage Sur Corpus, didactique, FLE.

Keywords : verbal interactions, multimodal corpus, data-driven learning, applied linguistics, French as a Foreign Language

\section{AUTEUR}

VIRGINIE ANDRÉ

ATILF - Université de Lorraine et CNRS 